\title{
$T$-cell response to adenovirus hexon and DNA-binding protein in mice
}

\author{
T McKelvey, A Tang, AJ Bett, DR Casimiro and M Chastain \\ Department of Viral Vaccine Research, Merck Research Laboratories, West Point, PA, USA
}

\begin{abstract}
The successful development of adenovirus vectors for vaccines and gene therapy will require a better understanding of the host immune response. Using the ELISPOT assay to measure IFN- $\gamma$-secreting $C D 8^{+}$cells, we identify immunodominant epitopes of the adenovirus hexon and $D N A$-binding protein in BALB/C and C57BL/6 mice. The $T$-cell response to the intramuscular administration of adenovirus serotype 5 peaks within a few weeks and gradually declines but is still detectable after 12 weeks. A second administration did not substantially increase the number of reactive $T$ cells. The $C D 8^{+} T$-cell response was
\end{abstract}

also similar between wild type and E1-deleted adenovirus. When B-cell-deficient mice were injected with adenovirus encoding the gene for secreted alkaline phosphatase, sera phosphatase activity was reduced more quickly in mice pre-exposed to adenovirus. These results add to the evidence that cell-mediated immunity is a substantial barrier to therapeutic adenoviral vectors and provide more quantitative tools to measure cellular immune responses to adenovirus.

Gene Therapy (2004) 11, 791-796. doi:10.1038/sj.gt.3302232

Published online 12 February 2004

Keywords: adenovirus; cell-mediated immunity; DNA-binding protein; ELISPOT; hexon

\section{Introduction}

Adenoviruses have been widely studied as gene-delivery vehicles for vaccines and gene therapy. Successful adenoviral infection and subsequent transgene expression has been demonstrated in a variety of species and target tissues. However, the usefulness of adenoviruses for the delivery of foreign genes is severely limited by the immune response of the host. ${ }^{1,2}$ Adenoviral vectors exhibit long-term expression of foreign genes in SCID or other immunocompromised mice, but in most studies with immunologically competent animals transgene expression diminishes over a period of weeks. ${ }^{3}$ The rapid disappearance of the desired protein reduces the utility of adenoviral vectors for treatment of chronic conditions and also as vaccines. This point has been further demonstrated by the failure of adenoviral vectors in gene therapy clinical trials.

Both humoral and cell-mediated responses are activated upon the administration of adenoviral vectors. ${ }^{4}$ The relative contribution of each depends on the host species, its MHC type, the vector backbone and the transgene. Some researchers have demonstrated that the majority of the host-directed immune response is against the transgene. ${ }^{5}$ Highly antigenic proteins such as nonself erythropoietin have been shown to be immunodominant. ${ }^{6}$ It is also well established that adenoviral proteins also serve as immunogens. Neutralizing antibodies to adenovirus are well documented in humans ${ }^{7}$ and mice. ${ }^{8}$ Of greater significance for gene therapy though is the elimination

Correspondence: M Chastain, Merck \& Co., 770 Sumneytown Pike, WP26-145, West Point, PA 19486, USA

Received 18 June 2003; accepted 20 November 2003; published online 12 February 2004 of adenovirus-transduced cells by cell-mediated immunity (CMI). In mice, $\mathrm{CD}^{+}$lymphocytes destroy adenovirusinfected cells in principal target tissues such as the liver, ${ }^{9}$ lung $^{10}$ and muscle. ${ }^{5}$ Whether a single administration of adenovirus is sufficient to generate cytotoxic T lymphocytes, or if the continued 'leaky' expression of virus proteins from E1-deleted vectors is required, is unclear. ${ }^{11,12}$

Several adenoviral proteins have been identified as the principal antigens for CMI. The structural proteins, which comprise the adenovirus capsid, the hexon, penton and fiber, have all been identified as containing MHC class Irestricted epitopes. ${ }^{13}$ The hexon protein, the most abundant capsid protein, is a strong stimulator in BALB/c $(\mathrm{H}-$ $2^{\mathrm{d})}$ mice $^{14}$ and also contains a conserved human CD4 ${ }^{+}$ epitope. ${ }^{15}$ In this report, we identify a hexon $\mathrm{H}-2^{\mathrm{d}}$ epitope by determining the number of IFN- $\gamma$-secreting cells with the ELISPOT assay. We also identify the DNA-binding protein, a nonstructural protein that is not packaged in the virion, as containing dominant $\mathrm{CD}^{+} \mathrm{T}$-cell epitopes in BALB/c and C57BL/6 $\left(\mathrm{H}-2^{\mathrm{b}}\right)$ mice.

Owing to the transient transgene expression, most adenovirus-vector gene therapy protocols require multiple administrations. Vectors that are effective with repeated dosing and can evade pre-existing immunity from natural infection will have to be developed. In this repot, we also examine the effect of pre-existing cellular, but not humoral immunity, on adenovirus delivery of a foreign gene in mice.

\section{Results}

Identification of adenovirus epitopes

The $\mathrm{CD}^{+}$epitopes of adenovirus type 5 hexon and DNA-binding protein were identified as shown in 
Figure 1 . The primary sequence of the hexon protein was divided into 24-mer peptides overlapping by 12 amino acids, which were then grouped into pools of 10 peptides and tested in the ELISPOT assay against mice vaccinated with wild-type Ad5 (wtAd5). The DNA-binding protein was divided into 22-mer peptides overlapping by 11 amino acids, which were also grouped into pools of 10 peptides and tested in the ELISPOT assay. Individual peptides from pools that scored positive were then tested in the assay against $\mathrm{CD}^{+}{ }^{+}$and $\mathrm{CD}^{+}{ }^{+}$-depleted splenocytes. In BALB/c mice, the hex21 24-mer peptide (amino acids 481-504, LPDKLKYSPSNVKISDNPNTYDYM) and the dbp43 22-mer peptide (amino acids 409-430, LGRQLPKLTPFALSNAEDLDAD) were identified to contain strong $\mathrm{CD}^{+}$epitopes. In C57BL/ 6 mice, the dbp43 22-mer peptide (amino acids 409-430, LGRQLPKLTPFALSNAEDLDAD) was identified to have the principal $\mathrm{CD}^{+}$epitope. Shorter 9-mer peptides (hex3: KYSPSNVKI, dbp7: LPKLTPFAL, dbp43: FALSNAEDL) were synthesized and tested for each of the three identified epitopes. Each of these peptides gave signals as high or higher than the longer peptides and was used for all subsequent assays.

\section{Variation of ELISPOT responses in individual mice}

ELISPOT assays were typically performed using spleens harvested from several mice and pooled to give an average signal. In order to determine the variability in the ELISPOT assay and the appropriate number of spleens to pool, we conducted ELISPOT assays on individual mice. BALB/c mice were vaccinated with
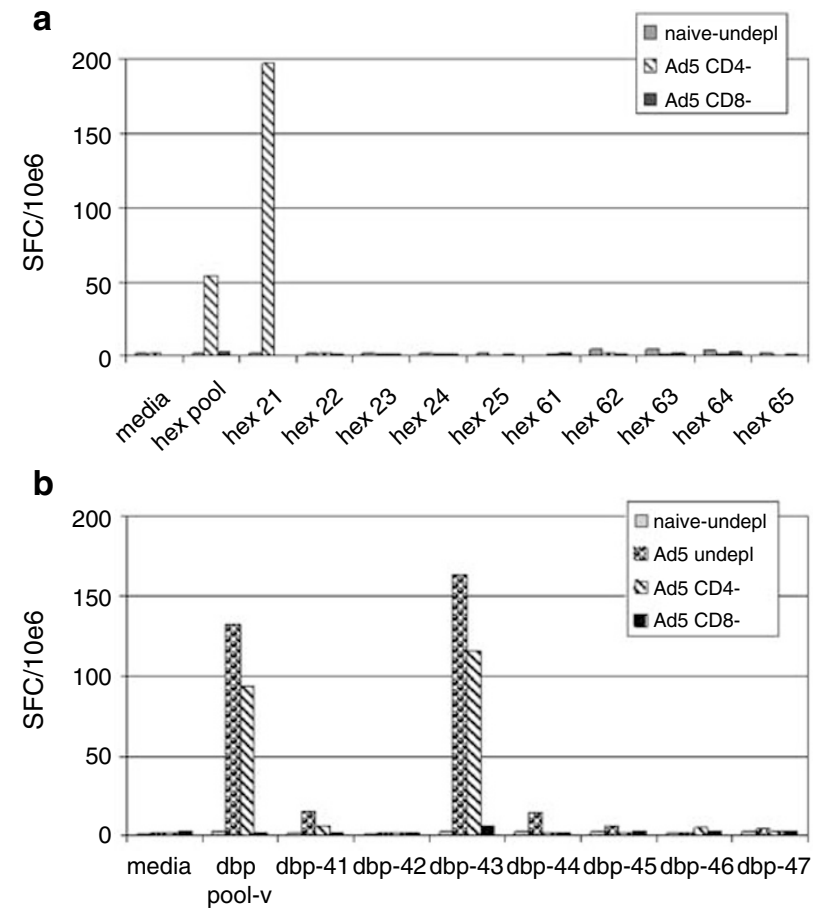

Figure 1 Identification of mouse $\mathrm{CD} 8^{+}$adenovirus protein epitopes. Mice were vaccinated with $10^{8}$ viral particles of wtAd5, then boosted 4 weeks later with the same dose. After 4 weeks, the spleens were harvested and the splenocytes were tested in an ELISPOT assay. The number of SFC per $10^{6}$ cells $\left(S F C / 10^{6}\right)$ tested for the positive peptides and the peptide pools in which they were screened are shown. (a) The hexon protein in BALB/c mice (DNA-binding protein not shown). (b) The DNA-binding protein in C57BL/6 mice.
$10^{8}$ viral particles of wtAd5 and 4 weeks later tested in the ELISPOT assay against the hex and dbp peptides. The results of 10 vaccinated mice and three unvaccinated mice tested are shown in Table 1 . In vaccinated mice, the SFC $/ 10^{6}$ ranged from 151 to 570 with an average of 310 and a s.d. of 130 for the hex3 peptide. Against the dbp7 peptide, the number of spot-forming cells per million ranged from 321 to 628 with an average of 456 and a s.d. of 111. Given the range of responses, we found it appropriate to conduct most assays using cells from three pooled spleens.

\section{Dose response of mice to adenovirus vaccination}

The magnitude of the $\mathrm{T}$-cell response to adenovirus vaccination was tested over a range of doses. BALB/c and C57BL/6 mice were injected with $10^{5}-10^{8}$ viral particles of wtAd5 and half the mice were boosted with the same dose 4 weeks later. The frequency of $\mathrm{T}$ cells secreting IFN- $\gamma$ in response to adenovirus peptides was determined in the ELISPOT assay 4 weeks later. As shown in Figure $2 \mathrm{a}$, the BALB/c mice showed an increasing number of IFN- $\gamma$-secreting cells responding to the $\mathrm{dbp}$ and hex peptides with increasing vaccination dose. Boosting added only a marginal increase in the signal at the time point analyzed. The C57BL/6 mice showed a similar pattern, however, the number of IFN- $\gamma$ secreting cells was lower (Figure $2 b$ ).

\section{Time course of ELISPOT response}

Additionally, we examined how the $\mathrm{CD}^{+}{ }^{+} \mathrm{T}$-cell response changed over time. Mice were primed with $10^{8}$ particles of Ad5 and 4 weeks later half the animals were boosted with the same dose. From 3 to 12 weeks later, the spleens were harvested and tested in the ELISPOT assay. In BALB/c mice, the ELISPOT response

Table 1 Variability of ELISPOT responses in individual mice

\begin{tabular}{lcccc}
\hline \multicolumn{5}{c}{ Spot number $10^{6}$ cells } \\
\hline$\#$ & Virus & Media & hex3 & $d b p 7$ \\
\hline 1 & 10e8 vp wtAd5 & 0 & 313 & 458 \\
2 & $\downarrow$ & 4 & 570 & 628 \\
3 & $\downarrow$ & 4 & 228 & 321 \\
4 & $\downarrow$ & 2 & 209 & 336 \\
5 & $\downarrow$ & 5 & 321 & 480 \\
6 & $\downarrow$ & 4 & 302 & 505 \\
7 & $\downarrow$ & 1 & 417 & 578 \\
8 & $\downarrow$ & 31 & 420 & 549 \\
9 & $\downarrow$ & 1 & 174 & 337 \\
10 & $\downarrow$ & 5 & 310 & 367 \\
& & 9 & 130 & 456 \\
Avg & & 2 & & 111 \\
s.d. & & 60 & 47 & 11 \\
11 & Naive & 62 & 9 & 6 \\
12 & $\downarrow$ & & & \\
13 & $\downarrow$ & 41 & 19 & 11 \\
Avg & & 34 & 25 & 5 \\
s.d. & & &
\end{tabular}

BALB/c mice were vaccinated with $10^{8}$ viral particles of wtAd5 and 4 weeks later tested individually in the ELISPOT assay. The number of $\mathrm{SFC} / 10^{6}$ for 10 vaccinated mice and three unvaccinated mice tested against the hex 3 and $\mathrm{dbp} 7$ peptides are shown. 


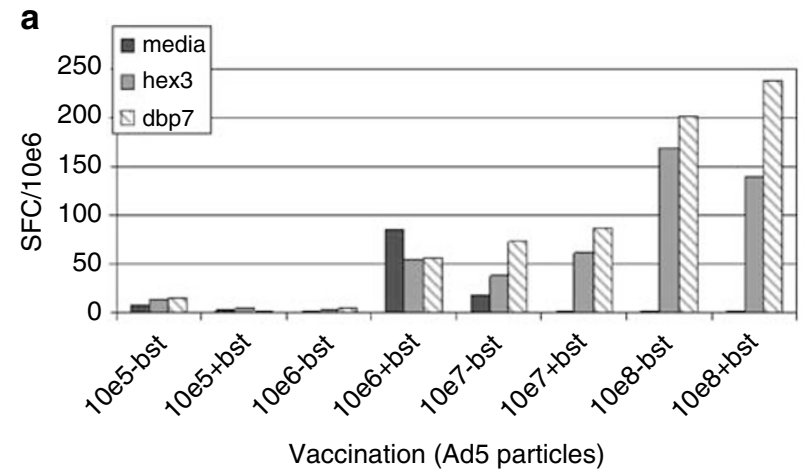

b

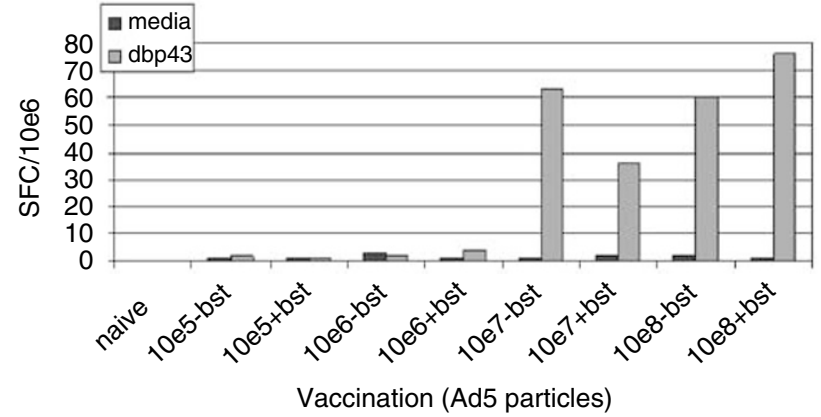

Figure 2 Dose response of mice to adenovirus vaccination. Mice were injected with $10^{5}-10^{8}$ viral particles of wtAd5. After 4 weeks, half were boosted with the same dose. After an additional 4 weeks, the cells from three spleens were pooled and the number of responding cells was determined in the ELISPOT assay. (a) BALB/c response to the hex3 and dbp7 peptides. (b) C57BL/6 response to the dbp43 peptide.

to the hex (Figure 3a) and dbp (Figure 3b) antigen was highest at the earliest time point tested and declined over the remaining time period. As seen previously, boosting did not give a consistent increase in the number of responder cells. A similar pattern in the immune responses was seen in C57BL/6 mice (Figure 3c). A second experiment (data not shown) in which the mice were primed (3-12 weeks prior to harvesting the spleens) or primed and boosted 4 weeks later (7-16 weeks prior to harvesting the spleens) also produced the same trend.

\section{Immune response of wild-type versus E1-deleted adenovirus}

Most of our studies were performed with wild-type Ad, whereas most of the gene therapy and vaccine studies are conducted using replication defective, E1 ${ }^{-}$adenovirus vectors. We compared wild-type and $\mathrm{E} 1^{-}$virus in the ELISPOT assay in order to examine the effect of the deletion of the E1 region on CMI. BALB/c and C57BL/6 mice were primed with $10^{8}$ viral particles of wtAd 5 or Ad5E1,- boosted 4 weeks later with the same vector, and tested $\mathrm{t} 3$ or 6 weeks later in ELISPOT assays. The results are displayed in Table 2. For both strains, deletion of the E1 region did not significantly change the T-cell responses.

\section{Functional role of $T$ cells in B-cell-deficient mice and pre-existing immunity}

We also investigated the functional role of $\mathrm{T}$ cells in the antiadeno immune response using adenovirus vectors encoding the secreted alkaline phosphatase (SEAP) gene.

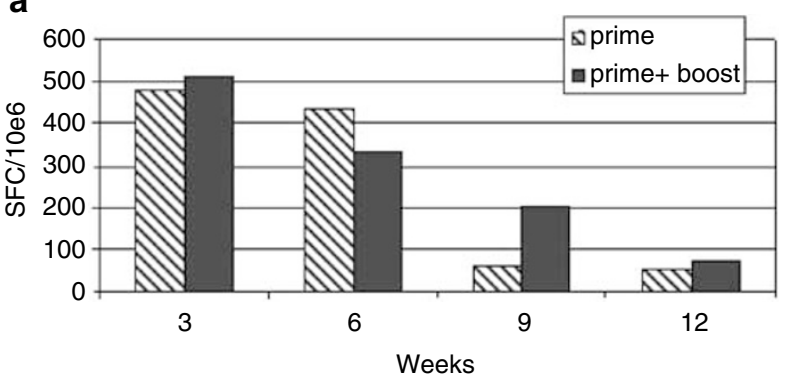

b
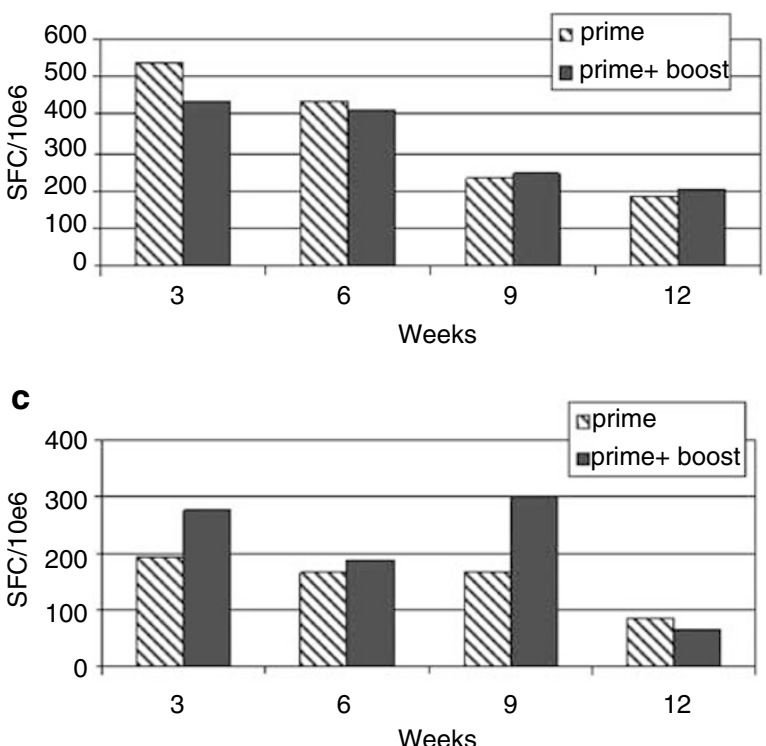

Figure 3 Time course of ELISPOT response. Mice were injected with $10^{8}$ viral particles of wtAd5. After 4 weeks, half were boosted with the same dose. After an additional 3-12 weeks, the cells from three spleens were pooled and the number of responding cells was determined in the ELISPOT assay. (a) $B A L B / c$ response to the hex 3 peptide. (b) $B A L B / c$ response to the dbp7 peptide. (c) C57BL/6 response to the dbp 43 peptide.

Table 2 Immune response of wild-type versus E1- adenovirus

\begin{tabular}{lccc}
\hline Antigen & Naive & wtAd5 & Ad5E1- \\
\hline $\begin{array}{l}\text { BALB/c-3 weeks postboost } \\
\text { Media }\end{array}$ & 10 & 13 & \\
hex3 & 17 & 132 & 60 \\
dbp7 & 21 & 158 & 128 \\
C57BL/6-6 weeks postboost & & & \\
Media & 47 & 18 & 2 \\
dbp43 & 14 & 380 & 329 \\
\hline
\end{tabular}

$\mathrm{BALB} / \mathrm{c}$ and C57BL/ 6 mice were primed with $10^{8}$ viral particles of wild type or Ad5E1- and boosted 4 weeks later with the same. After 3 or 6 weeks, the cells from three spleens were pooled and tested in the ELISPOT assay against hex or dbp peptides. Data from the $\mathrm{BALB} / \mathrm{c}$ mice is from 6 weeks postboost and is 3 weeks postboost for $\mathrm{C} 57 \mathrm{BL} / 6$ mice.

B-cell-deficient mice (Jh strain, $\mathrm{H}-2^{\mathrm{d}}$ ) were divided into three groups, which received either no injection, $10^{8}$ or $10^{9}$ viral particles of wtAd5. Sera from these mice were tested for Ad5 neutralization activity, and as expected for B-cell-deficient mice, no neutralization activity was 
detected (data not shown). In addition, some mice were killed and ELISPOT assays were conducted to determine the T-cell response to the hex and dbp peptides. The Jh mice injected with wtAd5 at the $10^{9}$ dose had signals of 210 and 157 IFN- $\gamma$-secreting cells per million total cells to hex 3 and $d b p 7$, respectively. The mice were injected with $10^{9}$ Ad5SEAP 8 weeks after the initial injection with wtAd5 and sera collected at days 2, 5, 8 and 13 . The levels of serum SEAP activity at these time points is shown in Figure 4a. Mice that had been previously injected with wtAd5 showed significantly reduced serum SEAP levels at all time points compared to the adenovirus naïve mice. The naive Jh mice had high serum SEAP activity for 8 days until declining to near background level at day 13.

In a second type of experiment to observe the effects of antiadenovirus T-cell immunity, SCID mice that lack B and $\mathrm{T}$ cells were injected with $10^{7}$ B-cell-depleted splenocytes from either naïve or wtAd5-primed BALB/ $c$ mice and then challenged with $10^{9}$ viral particles of Ad5SEAP. The serum SEAP activity over the next 34 days is shown in Figure $4 \mathrm{~b}$. The SEAP activity in control SCID mice remained unchanged over the 5 weeks examined, while the addition of primed antiadenovirus cells reduced serum SEAP activity to much lower levels over several weeks. The transfer of naïve B-cell-depleted
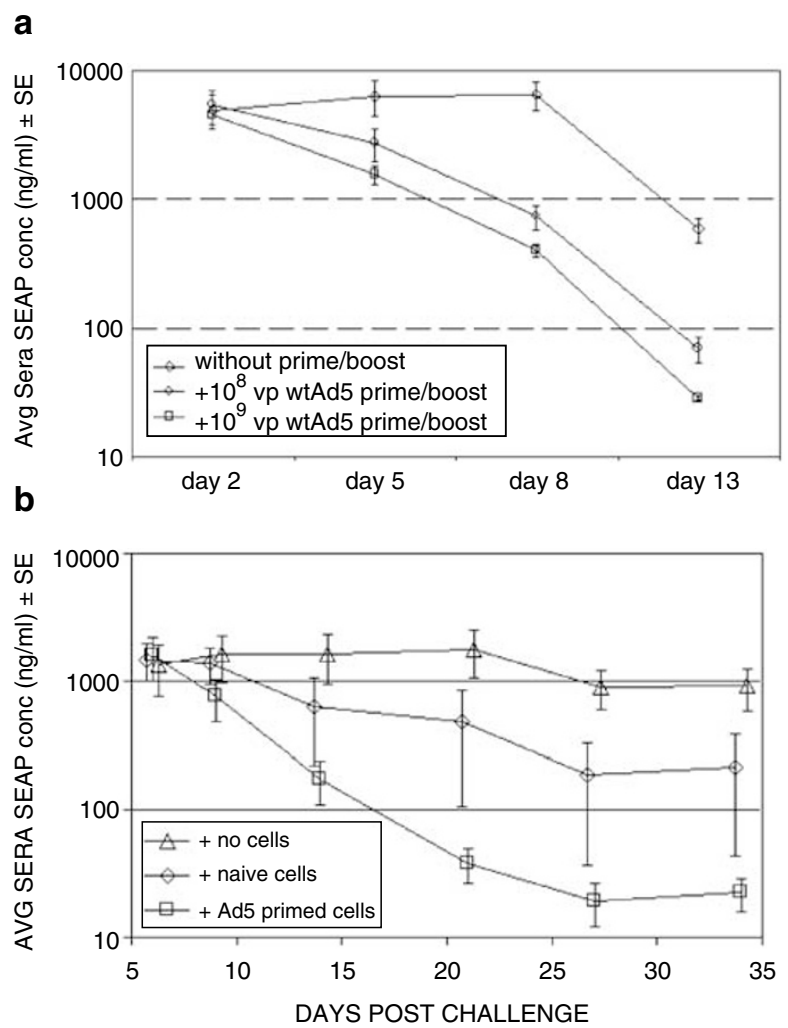

Figure 4 SEAP activity in immunodeficient mice. (a) Jh mice were primed with $10^{8}$ or $10^{9}$ viral particles of wt Ad5 and boosted with the same 3 weeks later. After an additional 3 weeks, the mice received $10^{9}$ viral particles of Ad5SEAP. At days 2, 5, 8 and 13 postinjection, the mice were bled and the sera analyzed for alkaline phosphatase activity (five mice per group). (b) Bcell-depleted splenocytes $\left(10^{7}\right)$ from untreated or Ad5-primed BALB/c mice $\left(10^{9}\right.$ virus particles wtAd5 4 weeks previously) were transferred by tail vein injection to SCID mice, which were challenged the next day with $10^{9}$ virus particles of Ad5SEAP. The serum alkaline phosphatase activity over the next 34 days is shown (10 mice per group). splenocytes also attenuated SEAP activity but at a slower rate than the primed cells. Pre-existing adenovirusspecific $T$ cells accelerate the attenuation of foreign gene expression in the absence of functional B cells.

\section{Discussion}

We demonstrate that BALB/c and C57BL/6 mice have strong cell-mediated immune responses to adenovirus serotype 5 proteins as determined with the ELISPOT IFN- $\gamma$ assay. BALB/c mice $\left(\mathrm{H}-2^{\mathrm{d}}\right)$ recognize $\mathrm{CD}^{+}$ epitopes in the adenovirus hexon and DNA-binding protein. $\mathrm{C} 57 \mathrm{BL} / 6$ mice $\left(\mathrm{H}-2^{\mathrm{b}}\right)$ mount a strong $\mathrm{CD} 8^{+}$ response against an epitope in the adenovirus DNAbinding protein. The identification of these $\mathrm{CD}^{+}$ peptide epitopes in adenovirus proteins enables the use of more quantitative T-cell assays such as ELISPOT and tetramer staining in gene therapy and vaccine studies using adenovirus vectors in mice. Previous work in mice found CTL responses to the hexon protein, but not to the DNA-binding protein. ${ }^{13}$ Possible reasons for the differences seen in the response to the DNA-binding protein include the source of antigen, and the nature of the assay. Our source of antigen was peptides, which may be more sensitive than the vaccinia virus encoding the entire DNA-binding protein as used in the previous study. Also, we used the ELISPOT assay that may be more sensitive than the CTL assays performed previously. In people, an immunodominant hexon $\mathrm{CD}^{+}$epitope has been previously defined in HLA-A2 donors, ${ }^{15}$ and adenovirus capsid proteins have previously been shown to be targets for cytotoxic T lymphocytes. ${ }^{16}$

We further characterized the magnitude and time course of the T-cell response in this model as measured in the ELISPOT assay. A dose-dependent increase in the number of IFN- $\gamma$ cells was seen over a range of $10^{5}-10^{8}$ viral particles. Higher doses did not induce a stronger ELISPOT response to the target peptides (data not shown). Also, the data did not show a consistent increase in the number of $\mathrm{CD}^{+} \mathrm{T}$ cells secreting interferon gamma by a prime/boost regimen over a single vaccination. The failure of repeated injections of adenovirus to boost the signal in the ELISPOT assay is surprising given that we have seen increases in ELISPOT signals to transgenes after repeated vaccinations in similar mouse experiments. The maximum number of IFN- $\gamma$-secreting cells occurred at the first time point tested ( 3 weeks), but was still detectable at 12 weeks. The smaller T-cell response seen in the ELISOPT 8 weeks after vaccination is still sufficient to impact subsequent gene expression by a heterologous adenovirus vector. As discussed below, in two different in vivo models using Jh or SCID mice, T-cell immunity after exposure to adenovirus reduces the duration of transgene expression. Additional assays will need to be developed to determine the ratio of memory versus effector $\mathrm{T}$ cells observed in the ELISPOT assay over time and their relative effects on subsequent adenovirus exposure.

The replication-defective adenovirus vectors used in gene therapy lack the E1 region, which activates transcription of the viral genome after infection. When we compared the T-cell stimulation induced by wildtype Ad5 and a replication-defective first-generation E1deleted vector, we found similar responses to the hexon 
and DNA-binding protein epitopes. Comparable CMI responses with these vectors would indicate that the immune response is being generated by the inoculum (without additional protein synthesis) ${ }^{12}$ or that there is some expression of viral proteins with both wild-type and virus. ${ }^{11}$ If the latter case is true, then future vectors will have to be more effective at preventing viral transcription. Vectors with deletions of the other earlytranscribed regions (E2, E3 and E4) and even 'gutless' vectors lacking all viral coding regions have been explored but have not yet been fully evaluated. ${ }^{17-19}$ The role of the E3 region in particular needs to be further defined as it contains immunomodulatory functions. ${ }^{20}$ Additional strategies being evaluated to attenuate the host antiadeno response include serotype switching ${ }^{21,22}$ and the coadministration of immunosuppressants. ${ }^{3,23-25}$

In addition to quantitating the $\mathrm{T}$-cell response to adenovirus epitopes in vitro using the ELISPOT assay, we developed a semiquantitative method to assess the strength of the T-cell response to adenovirus in vivo. The use of Ad5SEAP in Jh mice is a highly effective assay for measuring the effects of adenovirus T-cell responses in vivo. We compared the use of Jh mice to an adoptive transfer experiment in SCID mice similar to one previously reported. ${ }^{3}$ Both experiments showed that $\mathrm{T}$ cell immunity to adenovirus reduces subsequent gene expression, but the use of Jh mice is simpler since it does not require in vitro manipulation of the T cells.

In this paper, we have developed quantitative approaches for measuring anti-Ad5 cellular immunity in rodents. These approaches can likewise be extended to studies involving other adenovirus serotypes. Improved quantitation of these immune responses against the viral vector will provide valuable information in assessing the potency of Ad vector approaches for therapeutic and vaccine applications.

\section{Materials and methods}

\section{Animals}

Female BALB/c, C57BL/6, Jh and SCID mice were obtained from Taconic Laboratories at approximately 4-6 weeks old and given food and water ad lib. For i.m. vaccinations the mice were injected with $50 \mu \mathrm{l}$ into each rear quadriceps muscle. All experiments were approved by the Institutional Animal Care and Use Committee.

\section{Cell lines and viruses}

Cell culture media and reagents were obtained from Invitrogen Corp. unless stated otherwise. WtAd5 was obtained from the ATCC and propagated in Hela cells. The Hela cells were grown in monolayer in minimum essential medium, alpha medium supplemented with $100 \mathrm{U} / \mathrm{ml}$ penicillin, $100 \mu \mathrm{g} / \mathrm{ml}$ streptomycin, $4 \mathrm{mM} \mathrm{L}-$ glutamine and $10 \%(\mathrm{v} / \mathrm{v})$ UV-irradiated fetal bovine serum. AdE1- and Ad5SEAP were propagated in PER.C6 cells $^{26}$ grown in monolayer in William's Medium E Modified (Hyclone) supplemented as above. AdE1- and Ad5SEAP are replication-incompetent first generation vectors containing E1 region deletions from nt 342 to 3523 and in the case of Ad5SEAP an E3 deletion from nt 28133 to 30818 . In Ad5SEAP, the secreted alkaline phosphatase (SEAP) transgene ${ }^{27}$ is located in the E1 region in the E1 antiparallel orientation. Transcription of the transgene is driven by the human cytomegalovirus promoter including Intron $\mathrm{A}^{28}$ and terminated using the bovine growth hormone polyadenylation signal.

\section{Peptides}

Peptides were custom synthesized by Research Genetics.

\section{ELISPOT}

The ELISPOT assay was performed as previously descibed. ${ }^{29}$ Spleens were harvested from BALB/c, Jh or C57BL/ 6 mice, minced in K media (RPMI medium 1640 supplemented with $100 \mathrm{U} / \mathrm{ml}$ penicillin, $100 \mu \mathrm{g} / \mathrm{ml}$ streptomycin, $4 \mathrm{mM}$ L-glutamine, $55 \mu \mathrm{M} \beta$-mercaptoethanol, $10 \mathrm{mM}$ HEPES and 10\% fetal bovine serum) over a mesh insert and the RBCs lysed with AKC lysing buffer. The cells were diluted to $10^{7}$ cells $/ \mathrm{ml}$ in $\mathrm{K}$ media and $100 \mu \mathrm{l}$ of the cell suspension was added to $100 \mu \mathrm{l}$ of the appropriate antigen at $2 \mu \mathrm{g} / \mathrm{ml}$ in the well of a 96-well microplate with a nitrocellulose bottom coated with capture antibody (purified rat anti-mouse IFN- $\gamma$, PharMingen). The plates were incubated for $18-22 \mathrm{~h}$ at $37^{\circ} \mathrm{C}$ in $5 \% \mathrm{CO}_{2}$, washed and $100 \mu \mathrm{l} /$ well biotinylated rat antimouse IFN- $\gamma$ (PharMingen) was added. The plates were washed again and $100 \mu \mathrm{l} /$ well streptavidin-AP conjugate (PharMingen) was added. Color was developed by adding $100 \mu \mathrm{l} /$ well 1-STEP NBT-BCIP (Pierce) for 5-10 min. Spots were counted and the spot-forming cells (SFC) per $10^{6}$ cells were calculated. The nonspecific mitogen concanavalin A was the positive control and produced a solid color in all assays.

\section{Adoptive transfer}

Spleens from BALB/c mice were prepared as described above and subsequently depleted of B cells by magnetic separation according to the supplier's instructions using beads coated with an antibody to the mouse B220 antigen (Dynal Biotech). In total, $10^{7}$ B-cell-depleted splenocytes were injected via the tail vein into each SCID mouse. The following day the mice were challenged with $10^{9}$ virus particles of Ad5SEAP.

\section{SEAP assay}

Blood was collected from mice via the tail vein and sera collected after centrifugation. The sera was heat-treated for $30 \mathrm{~min}$ at $65^{\circ} \mathrm{C}$ to inactivate endogenous alkaline phosphatase activity and the secreted alkaline phosphatase activity measured using a Tropix Phospha-Light luminescence assay (Applied Biosystems). The light output over $5 \mathrm{~s}$ was measured on a Dynex MLX luminometer and relative luminescence output converted to $\mathrm{ng} / \mathrm{ml}$ of SEAP by linear regression of an alkaline phosphatase (Sigma) standard curve.

\section{References}

1 Yang Y, Li Q, Ertl HCJ, Wilson JM. Cellular and humoral immune responses to viral antigens create barriers to lungdirected gene therapy with recombinant adenoviruses. J Virol 1995; 69: 2004-2015.

2 Yei $S$ et al. Adenovirus-mediated gene transfer for cystic fibrosis: quantitative evaluation of repeated in vivo vector administration to the lung. Gene Therapy 1994; 1: 192-200.

3 Dai $\mathrm{Y}$ et al. Cellular and humoral immune responses to adenoviral vectors containing factor IX gene: tolerization of 
factor IX and vector antigens allows for long-term expression. Proc Natl Acad Sci USA 1995; 92: 1401-1405.

4 DeMatteo RP et al. Immunological barriers to hepatic adenoviral gene therapy for transplantation. Transplantation 1997; 63: 315-319.

5 Yang Y, Haecker S, Su Q, Wilson JM. Immunology of gene therapy with adenoviral vectors in mouse skeletal muscle. Hum Mol Gen 1996; 5: 1703-1712.

6 Tripathy SK, Black HB, Goldwasser E, Leiden JM. Immune responses to transgene-encoded proteins limit the stability of gene expression after injection of replication-defective adenovirus vectors. Nat Med 1996; 2: 545-550.

7 Gahery-Segard $\mathrm{H}$ et al. Immune response to recombinant capsid proteins of adenovirus in humans: antifiber and anti-penton base antibodies have a synergistic effect on neutralizing activity. J Virol 1998; 72: 2388-2397.

8 Chen P, Kovesdi I, Bruder JT. Effective repeat administration with adenovirus vectors to the muscle. Gene Therapy 2000; 7: 587-595.

9 Yang Y, Ertl HCJ, Wilson JM. MHC class I-restricted cytotoxic Tlymphocytes to viral antigens destroy hepatocytes in mice infected with E1-deleted recombinant adenoviruses. Immunity 1994; 1: 433-442.

10 Yang Y, Su Q, Wilson JM. Role of viral antigens in destructive cellular immune responses to adenovirus vector-transduced cells in mouse lungs. J Virol 1996; 70: 7209-7212.

11 Yang $\mathrm{Y}$ et al. Cellular immunity to viral antigens limits E1-deleted adenoviruses for gene therapy. Proc Natl Acad Sci USA 1994; 91: 4407-4411.

12 Kafri $\mathrm{T}$ et al. Cellular immune response to adenoviral vector infected cells does not require de novo viral gene expression: implications for gene therapy. Proc Natl Acad Sci USA 1998; 95: 11377-11382.

13 Jooss K, Ertl HCJ, Wilson J. Cytotoxic T-lymphocyte target proteins and their major histocampatibilty complex class I restriction in response to adenovirus vectors delivered to mouse liver. J Virol 1998; 72: 2945-2954.

14 Molinier-Frenkel V et al. Adenovirus hexon protein is a potent adjuvant for activation of a cellular immune response. J Virol 2002; 76: 127-135.

15 Olive $\mathrm{M}$ et al. The adenovirus capsid protein hexon contains a highly conserved human CD4+ T-cell epitope. Hum Gene Ther 2002; 13: 1167-1178.

16 Molinier-Frenkel $\mathrm{V}$ et al Immune response to recombinant adenovirus in humans: capsid proteins from viral input are targets for vector-specific cytotoxic T lymphocytes. J Virol 2000; 74 (16): 7678-7682.
17 Chirmule $\mathrm{N}$ et al. Role of E4 in eliciting CD4 T-cell and B-cell responses to adenovirus vectors delivered to murine and nonhuman primate lungs. J Virol 1998; 72: 6138-6145.

18 Lusky $\mathrm{M}$ et al. In vitro and in vivo biology of recombinant adenovirus vectors with E1, E1/E2A, or E1/E4 deleted. J Virol 1998; 72: 2022-2032.

19 Morsy $M$ et al An adenoviral vector deleted for all viral coding sequences results in enhanced safety and extended expression of a leptin transgene. Appl Biol Sci 1998; 95: 7866-7871.

20 Ilan $\mathrm{Y}$ et al. Insertion of the adenoviral E3 region into a recombinant viral vector prevents antiviral humoral and cellular immune responses and permits long-term gene expression. Proc Natl Acad Sci USA 1997; 94: 2587-2592.

21 Mastrangeli A et al. Sero-switch adenovirus-mediated in vivo gene transfer: circumvention of anti-adenovirus humoral immune defenses against repeat adenovirus vector administration by changing the adenovirus serotype. Hum Gene Ther 1996; 7: 79-87.

22 Mack CA et al. Circumvention of anti-adenovirus neutralizing immunity by administration of an adenoviral vector of an alternate serotype. Hum Gene Ther 1997; 8: 99-109.

23 Qin L et al. Adenovirus-mediated gene transfer of viral interleukin-10 inhibits the immune response to both alloantigen and adenoviral antigen. Hum Gene Ther 1997; 8: 1365-1374.

24 Peng Y, Falck-Pedersen E, Elkon KB. Soluble CD8 attenuates cytotoxic T-cell responses against replication-defective adenovirus affording transprotection of transgene in vivo. J Immun 2000; 165: 1470-1478.

25 Jooss K, Yang Y, Wilson J. Cyclophosphamide diminishes inflammation and prolongs transgene expression following delivery of adenoviral vectors to mouse liver and lung. Hum Gene Ther 1996; 7: 1555-1566.

26 Fallaux FJ et al New helper cells and matched early region 1-deleted adenovirus vectors prevent generation of replicationcompetent adenoviruses. Hum Gene Ther 1998; 9: 1909-1917.

27 Berger J et al. Secreted placental alkaline phosphatase: a powerful new quantitative indicator of gene expression in eukaryotic cells. Gene 1988; 66: 1-10.

28 Chapman B, Thayer R, Vincent K, Haigwood N. Effect of intron A from human cytomegalovirus (Towne) immediate-early gene on heterologous expression in mammalian cells. Nucleic Acids Res 1991; 19: 3979-3986.

29 Klinman DM, Nutman TB. ELISPOT Assay to detect cytokinesecreting murine and human cells. In: Coligan JE et al (ed). Current Protocols in Immunology. John Wiley \& Sons, Inc: New York, 1994; 1:pp 6.19.1-8. 